\title{
Fitness Estimation Based Particle Swarm Optimization Algorithm for Layout Design of Truss Structures
}

\author{
Ayang Xiao, ${ }^{1}$ Benli Wang, ${ }^{1}$ Chaoli Sun, ${ }^{2}$ Shijie Zhang, ${ }^{1}$ and Zhenguo Yang ${ }^{1}$ \\ ${ }^{1}$ Research Centre of Satellite Technology, Harbin Institute of Technology, Harbin 150001, China \\ ${ }^{2}$ Complex System and Computational Intelligence Laboratory, Taiyuan University of Science and Technology, Taiyuan 030024, China \\ Correspondence should be addressed to Ayang Xiao; ayang.xiao@gmail.com
}

Received 2 August 2014; Revised 8 September 2014; Accepted 9 September 2014; Published 29 September 2014

Academic Editor: Shifei Ding

Copyright (c) 2014 Ayang Xiao et al. This is an open access article distributed under the Creative Commons Attribution License, which permits unrestricted use, distribution, and reproduction in any medium, provided the original work is properly cited.

\begin{abstract}
Due to the fact that vastly different variables and constraints are simultaneously considered, truss layout optimization is a typical difficult constrained mixed-integer nonlinear program. Moreover, the computational cost of truss analysis is often quite expensive. In this paper, a novel fitness estimation based particle swarm optimization algorithm with an adaptive penalty function approach (FEPSO-AP) is proposed to handle this problem. FEPSO-AP adopts a special fitness estimate strategy to evaluate the similar particles in the current population, with the purpose to reduce the computational cost. Further more, a laconic adaptive penalty function is employed by FEPSO-AP, which can handle multiple constraints effectively by making good use of historical iteration information. Four benchmark examples with fixed topologies and up to 44 design dimensions were studied to verify the generality and efficiency of the proposed algorithm. Numerical results of the present work compared with results of other state-of-theart hybrid algorithms shown in the literature demonstrate that the convergence rate and the solution quality of FEPSO-AP are essentially competitive.
\end{abstract}

\section{Introduction}

As a typical real world project, truss structural analysis is considered to be computationally expensive [1]. Moreover, truss layout optimization, which solves truss sizing variables (e.g., cross-sectional areas of elements) and shape variables (e.g., coordinates of nodes) simultaneously, is known as a typical multimodal and highly nonlinear problem [2]. The various differences (e.g., physical nature, magnitude, continuity, etc.) between the two types of variables make the truss layout optimization problem a difficult task [3]. Hence, in the past decades, researchers mainly focused on solving truss layout optimization via multilevel methods [4].

Vanderplaats and Moses [5] proposed an alternating gradient method to decompose truss layout optimization into a number of subproblems, each of which optimized a subset of the design variables. The subproblems are solved iteratively until a converged optimal solution is found. Zhou [6] used a similar two-level approximation concept to optimize the cross-sectional areas of the members and the coordinates of the joints. Gil and Andreu [7] used fully stressed design method and conjugate gradient method to optimize the sizing and shape parameters of bridges, respectively.

Due to the strong coupling between the variables, the search efficiency of multilevel methods is often limited [8]. Aiming for addressing this issue and also benefiting from recent rapid advances in computational power, single-level methods which optimize all design variables simultaneously are becoming popular and competitive. Wang et al. [9] presented an optimality criteria (OC) algorithm for spatial truss layout optimization. Fourie and Groenwold [10] used new operators, namely, the elite velocity and the elite particle, in the standard particle swarm optimization (PSO) algorithm to optimize the truss layout, with the purpose of increasing the probability of migration to regions with high fitness. In fact, various metaheuristic algorithms including simulated annealing (SA) [11], genetic algorithm (GA) [12], charged system search (CSS) [13], and artificial bee colony algorithm $(\mathrm{ABC})[14]$ have been introduced to address truss layout optimization problems. 
More recently, researchers turn to hybridizing different techniques to further enhance the searching efficiency of metaheuristic algorithms. Lingyun et al. [18] proposed a niche hybrid genetic algorithm to solve the truss shape and sizing optimization in a simple and effective manner. Kaveh and Zolghadr [23] developed a hybridized CSS-BBBC algorithm with trap recognition capability for truss layout optimization. Zuo et al. [24] proposed a hybrid OC-GA approach for fast and global truss layout optimization; Kaveh and Javadi [25] used harmony search and ray optimizer to enhance the PSO algorithm to optimize truss layout under multiple frequency constraints; Liu and Ye [26] designed a genetic simulated annealing algorithm for domes layout optimization. Gholizadeh [17] proposed a hybridized cellular automata and PSO algorithm for truss layout optimization.

However, the common weakness of metaheuristic algorithms based structural optimization is that a huge number of structural analyses are required, which is quite time-consuming. In this paper, we propose a new hybridized algorithm, termed FEPSO-AP for truss layout optimization that aims to enhance the optimal efficiency by using the fitness estimations to partly substitute the computationally expensive fitness calculations. The finite element method (FEM) is adopted to evaluate the structural performance. Empirical results demonstrate that the proposed method is highly promising for truss layout optimization.

\section{Statement of Truss Layout Optimization Problem}

The main aim of truss layout optimization can be formulated as follows:

$$
\begin{array}{ll}
\text { Minimize: } & w(\mathbf{X})=\sum_{i=1}^{n e} \rho_{i} \cdot A_{i} \cdot l_{i}, \\
\text { Subject to: } & g(\mathbf{X}) \leq 0,
\end{array}
$$

where $w$ and $g$ denote the weight of truss structure and the maximum violated structural constraint; $\mathbf{X}$ is the vector of all design variables; $\rho_{i}, l_{i}$, and $A_{i}$ represent the material density, length, and cross-sectional area of the $i$ th truss element, respectively.

Different types of constraints might be considered simultaneously depending on the problem to be solved. Four typical design constraints involved in this work can be stated by

$$
\begin{gathered}
g_{i}^{\sigma}(\mathbf{X})=\frac{\sigma_{i}(\mathbf{X})}{\sigma_{i, \mathrm{all}}}-1 \leq 0, \quad i=1,2, \ldots, n e, \\
g_{j}^{d}(\mathbf{X})=\frac{d_{j}(\mathbf{X})}{d_{j, \mathrm{all}}}-1 \leq 0, \quad j=1,2, \ldots, n j, \\
g_{i}^{b}(\mathbf{X})=\frac{\left|\sigma_{i}(\mathbf{X})\right|}{\sigma_{i, e r}}-1 \leq 0, \quad \text { if } \sigma_{i}(\mathbf{X})<0, \\
g_{k}^{f}(\mathbf{X})=\frac{f_{k}(\mathbf{X})}{f_{k, \mathrm{all}}}-1 \leq 0, \quad k=1,2, \ldots, n k,
\end{gathered}
$$

in which $g_{i}^{\sigma}$ is the truss element stress constraint, while $\sigma_{i}$ and $\sigma_{i, \text { all }}$ stand for the actual largest stress of the $i$ th truss element and its stress limit, respectively; $g_{j}^{d}$ is the nodal displacement constraint, while $d_{j}$ and $d_{j, \text { all }}$ stand for the actual largest nodal displacement of the $j$ th node and its displacement limit, respectively; $g_{i}^{b}$ is the local buckling constraint, while $\sigma_{i, e r}$ stands for the Euler critical stress of the $i$ th element; $g_{k}^{f}$ is the structural natural frequency constraint, while $f_{k}$ and $f_{k \text {,all }}$ stand for the $k$ th structural natural frequency and its frequency limit; $n e, n j$, and $n k$ represent the number of structural elements, nodes, and constrained natural frequencies, respectively.

\section{FEPSO-AP Algorithm}

This section describes the fitness estimation based PSO algorithm with an adaptive penalty function approach (FEPSOAP) developed in this research. As FEPSO-AP integrates PSO, $\mathrm{FE}$, and $\mathrm{AP}$, this section recalls the basic concept of PSO algorithm, fitness estimation strategy, and adaptive constraint handling approach. Finally, a framework of FEPSO-AP algorithm is presented.

3.1. The PSO Algorithm. As one of the most popular metaheuristic algorithms, PSO has found a wide application in real world projects for its structural concision and searching efficiency [17]. In a standard PSO [27], it is assumed that each of the particles has a position and a certain velocity. The position of particle represents a candidate solution to the optimization problem, and the velocity of particle determines the particle's movement. Hence, the flying of particles can be considered as the swarm searching of design domain.

If the particle flies from its current position to the next position, its velocity and position are updated by

$$
\begin{gathered}
\vec{v}_{i}(t+1)=\omega \vec{v}_{i}(t)+c_{1} \mathbf{r}_{1}\left(\vec{p}_{i}(t)-\vec{x}_{i}(t)\right) \\
+c_{2} \mathbf{r}_{2}\left(\vec{p}_{g}(t)-\vec{x}_{i}(t)\right), \\
\vec{x}_{i}(t+1)=\vec{x}_{i}(t)+\vec{v}_{i}(t+1),
\end{gathered}
$$

in which $\vec{v}_{i}(t)$ and $\vec{x}_{i}(t)$ indicate the velocity and position of the $i$ th particle at iteration $t ; \vec{p}_{i}(t)$ and $\vec{p}_{g}(t)$ represent the historical best position of the $i$ th particle and the global best position of all particles till iteration $t$, respectively. $\omega$ stands for the inertia weight; $c_{1}$ and $c_{2}$ are cognitive and social parameters, respectively. $\mathbf{r}_{1}$ and $\mathbf{r}_{2}$ are diagonal matrixes, of which the diagonal elements are uniformly distributed random numbers among the range of $(0,1)$.

3.2. The Fitness Estimation Strategy. Sun et al. [28] proposed a novel fitness estimation strategy for PSO to solve computationally expensive problems.

According to (3), the positions of any two arbitrary particles selected from the swarm can be formulated by

$$
\begin{aligned}
\vec{x}_{i}(t+1)= & \left(1+\omega-\varphi_{1}-\varphi_{2}\right) \vec{x}_{i}(t)+\varphi_{1} \vec{p}_{i}(t) \\
& +\varphi_{2} \vec{p}_{g}(t)-\omega \vec{x}_{i}(t-1),
\end{aligned}
$$




$$
\begin{aligned}
\vec{x}_{j}(t+1)= & \left(1+\omega-\varphi_{1}^{\prime}-\varphi_{2}^{\prime}\right) \vec{x}_{j}(t)+\varphi_{1}^{\prime} \vec{p}_{j}(t) \\
& +\varphi_{2}^{\prime} \vec{p}_{g}(t)-\omega \vec{x}_{j}(t-1)
\end{aligned}
$$

in which $\varphi_{1}=c_{1} \mathbf{r}_{1}, \varphi_{2}=c_{2} \mathbf{r}_{2}, \varphi_{1}^{\prime}=c_{1} \mathbf{r}_{1}^{\prime}$, and $\varphi_{2}^{\prime}=c_{2} \mathbf{r}_{2}^{\prime}$. It can be found that the above two equations are both related to the global best position $\vec{p}_{g}(t)$. Hence, (4) can be abbreviated to

$$
\begin{aligned}
\varphi_{2} \vec{x}_{j}(t+1)+\varphi_{2}^{\prime}\left(1+\omega-\varphi_{1}-\varphi_{2}\right) \vec{x}_{i}(t) \\
+\varphi_{2}^{\prime} \varphi_{1} \vec{p}_{i}(t)+\varphi_{2} \omega \vec{x}_{j}(t-1) \\
=\varphi_{2}^{\prime} \vec{x}_{i}(t+1)+\varphi_{2}\left(1+\omega-\varphi_{1}^{\prime}-\varphi_{2}^{\prime}\right) \vec{x}_{j}(t) \\
\quad+\varphi_{2} \varphi_{1}^{\prime} \vec{p}_{j}(t)+\varphi_{2}^{\prime} \omega \vec{x}_{i}(t-1) .
\end{aligned}
$$

Use a virtual position $\vec{x}_{v}(t+1)$ to present the value of (5). Supposing the influences of all coefficients are similar, Figure 1 describes the geometrical relationship among the virtual position $\vec{x}_{\nu}(t+1)$ and all subitems contained by (5). As shown in Figure 1, the fitness of virtual position $\vec{x}_{v}(t+1)$ can be estimated by either the fitness of $\vec{x}_{j}(t+1), \vec{x}_{i}(t)$, $\vec{p}_{i}(t)$, and $\vec{x}_{j}(t-1)$ or the fitness of $\vec{x}_{i}(t+1), \vec{x}_{j}(t), \vec{p}_{j}(t)$, and $\vec{x}_{i}(t-1)$. Hence, if the current fitness value and the historical best fitness of every particle at iteration $t$ and $t-1$ are obtained already, the explicit relationship between the fitness of particle $i$ and $j$ at iteration $t+1$ can be expressed in the following formulations:

$$
\begin{aligned}
& f^{e}\left(\vec{x}_{j}(t+1)\right) \\
& =d_{v}^{j}(t+1) \\
& \cdot\left\{\alpha \cdot \left[\frac{f\left(\vec{x}_{i}(t+1)\right)}{d_{v}^{i}(t+1)}+\frac{f\left(\vec{x}_{i}(t-1)\right)}{d_{v}^{i}(t-1)}\right.\right. \\
& \left.+\frac{f\left(\vec{x}_{j}(t)\right)}{d_{v}^{j}(t)}+\frac{f\left(\vec{p}_{j}(t)\right)}{d_{v}^{p j}(t)}\right] \\
& \left.-\frac{f\left(\vec{x}_{j}(t-1)\right)}{d_{v}^{j}(t-1)}+\frac{f\left(\vec{x}_{i}(t)\right)}{d_{v}^{i}(t)}+\frac{f\left(\vec{p}_{i}(t)\right)}{d_{v}^{p i}(t)}\right\},
\end{aligned}
$$

$\alpha$

$$
=\frac{\left(1 / d_{v}^{j}(t+1)\right)+\left(1 / d_{v}^{j}(t-1)\right)+\left(1 / d_{v}^{i}(t)\right)+\left(1 / d_{v}^{p i}(t)\right)}{\left(1 / d_{v}^{i}(t+1)\right)+\left(1 / d_{v}^{i}(t-1)\right)+\left(1 / d_{v}^{j}(t)\right)+\left(1 / d_{v}^{p j}(t)\right)},
$$

in which $d_{v}^{i}(t+1), d_{v}^{i}(t), d_{v}^{i}(t-1), d_{v}^{j}(t+1), d_{v}^{j}(t), d_{v}^{j}(t-1)$, $d_{v}^{p i}(t)$, and $d_{v}^{p j}(t)$ represent the distance between the virtual position $\vec{x}_{v}(t+1)$ and the real positions $\vec{x}_{i}(t+1), \vec{x}_{i}(t), \vec{x}_{i}(t-1)$, $\vec{x}_{j}(t+1), \vec{x}_{j}(t), \vec{x}_{j}(t-1), \vec{p}_{i}(t)$, and $\vec{p}_{j}(t)$, respectively.

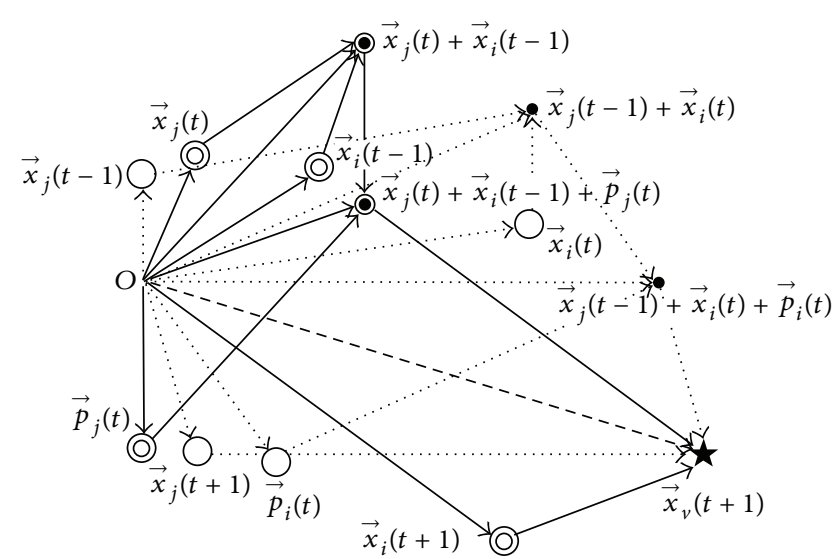

FIGURE 1: Illustration of the virtual position.

3.3. Adaptive Constraint Handling Approach. As mentioned in Section 2, truss layout optimization problem is often taken as a multiple constrained optimization problem. Coello Coello [29] pointed out that the constraint handling procedure plays an important role in the exploration and exploitation of constrained optimization problems. Similar to other metaheuristic algorithms, FEPSO is designed for unconstrained problems. Hence, it is necessary to incorporate constraint handling approach into FEPSO to solve truss layout optimization problems.

In this work, we use penalty function methods (PEM) to transfer a constrained problem into an unconstrained problem by adding the influence of violated constraints to the initial fitness function. A pseudoobjective function is stated as follows:

$$
\Phi(\mathbf{X})=f(\mathbf{X})+W \times G(\mathbf{X})
$$

where $W$ and $G(\mathbf{X})$ are positive penalty factor and penalty function. The common difficulty existing in standard PEM is to set the constant penalty factor $W$ properly. Runarsson and Yao [30] pointed out that if the penalty factor $W$ turns out to be too large, the searching landscape would be quite rough, and thus qualified exploitation and exploration of the design domain are hard to be achieved; on the contrary, if the penalty factor $W$ is too small, it would be quite possible to lose feasible solutions.

As summarized in [29], the common target of adaptive penalty factors is to make a good balance between the objective function and the constraints violation. In this work, we developed a laconic adaptive penalty factor as follows:

$$
W=2^{1-\rho}
$$

in which $\rho$ is the ratio of feasible solutions in current population. It can be concluded that if $W$ is too small, the obtained unfeasible solutions will increase the value of $\rho$, and thus, the algorithm would be pushed to explore more feasible solutions. On the other hand, if the feasible solutions congregate in the population, the value of $W$ will approach zero, and thus a detailed exploitation would be performed. 
3.4. The Framework of FEPSO-AP Algorithm. The penalty function $G(\mathbf{X})$ of truss layout optimization problem is determined by

$$
\begin{aligned}
& G(\mathbf{X})=w(\mathbf{X}) \times V(\mathbf{X}), \\
& V(\mathbf{X})=\max \{0, g(\mathbf{X})\},
\end{aligned}
$$

in which $\mathbf{X}$ is the structural design vector, $V(\mathbf{X})$ is the violated constraints, and $w(\mathbf{X})$ and $g(\mathbf{X})$ are defined by (1).

According to (8)-(10), the pseudoobjective function can be abbreviated to

$$
\Phi(\mathbf{X})=w(\mathbf{X}) \times\left(1+2^{1-\rho} \times V(\mathbf{X})\right) .
$$

To transfer the formulated truss layout optimization problem into an unconstrained optimization problem, the adaptive penalty function, the fitness estimation strategy, and the PSO algorithm are assembled as follows.

Step 1. Set the termination condition of optimization and the initial values of $\omega, c_{1}$, and $c_{2}$. Set the iteration times iter $=1$.

Step 2. Generate the initial positions $\mathbf{X}=\left\{\vec{x}_{i}(t)\right\}$ and the initial velocities $\mathbf{V}=\left\{\vec{v}_{i}(t)\right\}$ of particles population randomly, $i=1,2,3, \ldots$, popsize.

Step 3. Calculate the fitness values $f\left(\vec{x}_{i}(t)\right)$ of all particles and update every particle's historical best position $\vec{p}_{i}(t)$ and the global best position $\vec{p}_{g}(t)$ by

$$
\begin{gathered}
f\left(\vec{x}_{i}(t)\right)=\Phi\left(\vec{x}_{i}(t)\right), \\
\vec{p}_{i}(t)=\min \left\{f\left(\vec{x}_{i}\left(t^{\prime}\right)\right)\right\}, \quad t^{\prime}=1,2,3, \ldots, t, \\
\vec{p}_{g}(t)=\min \left\{f\left(\vec{x}_{i}(t)\right)\right\}, \quad i=1,2,3, \ldots, \text { popsize. }
\end{gathered}
$$

Step 4. Update the velocity $\vec{v}_{i}(t+1)$ and the position $\vec{x}_{i}(t+1)$ of every particle based on (3). Set the iteration times iter $=$ iter +1 .

Step 5. Calculate the distances between every pair of particles.

(5.1) Select particle $i$ randomly and calculate its fitness value $f\left(\vec{x}_{i}(t+1)\right)$ based on (13).

(5.2) Choose a particle $j$ nearby particle $i$ and calculate the virtual position $\vec{x}_{v}(t+1)$ by using the historical information of particle $j$ based on (5).

(5.3) If $\vec{x}_{v}(t+1)$ does not coincide with $\vec{x}_{i}(t+1), \vec{x}_{j}(t)$, $\vec{p}_{j}(t), \vec{x}_{i}(t-1), \vec{x}_{j}(t+1), \vec{x}_{i}(t), \vec{p}_{i}(t)$, or $\vec{x}_{j}(t-1)$, estimate the fitness value $f^{e}\left(\vec{x}_{j}(t+1)\right)$ of particle $j$ by (6); otherwise, calculate the fitness value of particle $j$ based on (13).

(5.4) Repeat (5.1)-(5.4) till the fitness values of all particles are evaluated.

Step 6. Update every particle's historical best position $\vec{p}_{i}(t+1)$ and the global best position $\vec{p}_{g}(t+1)$. If $\vec{p}_{g}(t+1)$ is obtained by estimation, recalculate the corresponding particle's fitness value by (13), and rechoose the global best position $\vec{p}_{g}(t+1)$.
TABLE 1: Loading condition acting on the planar 15-bar truss.

\begin{tabular}{lccc}
\hline Case & Node & $F_{x}$ (kips) & $F_{y}$ (kips) \\
\hline 1 & 8 & 0 & -10.0 \\
\hline
\end{tabular}

Repeat this step till a nonestimated value of $\vec{p}_{g}(t+1)$ is obtained.

Step 7. Repeat Steps 4-6 till the termination condition is reached. Output the global best position $\vec{p}_{g}(t+1)$ and its objective value.

\section{Benchmark Examples}

The following four benchmark examples have been used to demonstrate the generality and efficiency of the FEPSO-AP algorithm:

(i) a planar 15-bar truss subjected to a single load condition and stress constraints,

(ii) a spatial 25-bar truss subjected to a single load condition under stress and displacement constraints,

(iii) a planar 37-bar truss subjected to multiple frequency constraints,

(iv) a planar 47-bar truss subjected to three load conditions under stress and local buckling constraints.

Programs of FEPSO-AP algorithm and structural finite element method (FEM) algorithm are developed by using MATLAB R2013a. A personal computer with a Pentium E5700 processor and $2 \mathrm{~GB}$ memory under the Microsoft Windows 7 operating system has been used to run the optimization software.

For all benchmarks examined in this study, the FEPSOAP algorithm parameters are set as the usual constants of standard PSO which are obtained by [31]: $\omega=0.7298, c_{1}=$ 2.05 , and $c_{2}=2.05$. According to the design dimensions of four benchmarks, the population sizes are set as $46,26,38$, and 88 , while the maximum numbers of FEM analyses are set as $4000,4500,8000$, and 20000 , respectively.

Twenty-five independent runs are performed with the best one being selected for each problem.

4.1. Planar 15-Bar Truss. The original geometry of planar 15bar truss is shown in Figure 2(a). Two nodes (ID: 1 and 5) are totally fixed and the $x$-coordinates of other two nodes (ID: 4 and 8 ) are fixed as well. The single loading condition is listed in Table 1.

All design variables are classified into 23 groups:

$$
\begin{aligned}
& \text { sizing variables: } A_{i}, i=1,2, \ldots, 15 ; \\
& \text { shape variables: } x_{2}=x_{6} ; x_{3}=x_{7} ; y_{2} ; y_{3} ; y_{4} ; y_{6} ; y_{7} \text {; } \\
& y_{8} \text {. }
\end{aligned}
$$

Material parameters and design constraints are listed in Table 2 .

Figure 2(b) shows the optimum design found by this work, of which two nodes (ID: 4 and 8) are highly overlapped. 
TABLE 2: Material parameters, design constraints, and search range of the planar 15-bar truss optimization problem.

\begin{tabular}{|c|c|}
\hline Category & Values \\
\hline \multicolumn{2}{|l|}{ Material Parameters } \\
\hline Density & $0.1 \mathrm{lb} / \mathrm{in}^{3}$ \\
\hline Modulus of elasticity & $1 \times 10^{4} \mathrm{ksi}$ \\
\hline \multicolumn{2}{|l|}{ Constraints } \\
\hline Stress & The allowable elements stress interval: [ $-25 \mathrm{ksi}, 25 \mathrm{ksi}]$ \\
\hline \multicolumn{2}{|l|}{ Search range } \\
\hline Shape variables & $\begin{array}{l}100 \text { in. } \leq x_{2} \leq 140 \text { in.; } 220 \text { in. } \leq x_{3} \leq 260 \text { in.; } 100 \text { in. } \leq y_{2} \leq 140 \text { in.; } 100 \text { in. } \leq y_{3} \leq 140 \text { in.; } \\
50 \text { in. } \leq y_{4} \leq 90 \text { in.; }-20 \text { in. } \leq y_{6} \leq 20 \text { in.; }-20 \text { in. } \leq y_{7} \leq 20 \text { in.; } 20 \text { in. } \leq y_{8} \leq 60 \text { in.; } x_{1,5}=0 \text { in.; } \\
y_{1}=120 \text { in.; } x_{4,8}=360 \text { in. }\end{array}$ \\
\hline Sizing variables & $\begin{array}{l}S=\{0.111,0.141,0.174,0.220,0.270,0.287,0.347,0.440,0.539,0.954,1.081,1.174,1.333,1.488,1.764,2.142, \\
2.697,2.800,3.131,3.565,3.813,4.805,5.952,6.572,7.192,8.525,9.300,10.850,13.330,14.290,17.170, \\
19.180\} \text { in. }^{2} \\
A_{i} \in S, \quad i=1,2, \ldots, 15\end{array}$ \\
\hline
\end{tabular}

TABle 3: Comparison of optimized designs found for the planar 15-bar truss.

\begin{tabular}{|c|c|c|c|c|c|c|c|}
\hline No. & Variable & FA [15] & FM-GA [16] & PSO [17] & CPSO [17] & SCPSO [17] & FEPSO-AP \\
\hline 1 & $A_{1}$ & 0.954 & 1.081 & 0.954 & 1.174 & 0.954 & 1.081 \\
\hline 2 & $A_{2}$ & 0.539 & 0.539 & 1.081 & 0.539 & 0.539 & 0.539 \\
\hline 3 & $A_{3}$ & 0.220 & 0.287 & 0.270 & 0.347 & 0.270 & 0.270 \\
\hline 4 & $A_{4}$ & 0.954 & 0.954 & 1.081 & 0.954 & 0.954 & 0.954 \\
\hline 5 & $A_{5}$ & 0.539 & 0.539 & 0.539 & 0.954 & 0.539 & 0.539 \\
\hline 6 & $A_{6}$ & 0.220 & 0.141 & 0.287 & 0.141 & 0.174 & 0.111 \\
\hline 7 & $A_{7}$ & 0.111 & 0.111 & 0.141 & 0.141 & 0.111 & 0.111 \\
\hline 8 & $A_{8}$ & 0.111 & 0.111 & 0.111 & 0.111 & 0.111 & 0.111 \\
\hline 9 & $A_{9}$ & 0.287 & 0.539 & 0.347 & 1.174 & 0.287 & 0.347 \\
\hline 10 & $A_{10}$ & 0.440 & 0.440 & 0.440 & 0.141 & 0.347 & 0.347 \\
\hline 11 & $A_{11}$ & 0.440 & 0.539 & 0.270 & 0.440 & 0.347 & 0.440 \\
\hline 12 & $A_{12}$ & 0.220 & 0.270 & 0.111 & 0.440 & 0.220 & 0.287 \\
\hline 13 & $A_{13}$ & 0.220 & 0.220 & 0.347 & 0.141 & 0.220 & 0.287 \\
\hline 14 & $A_{14}$ & 0.270 & 0.141 & 0.440 & 0.141 & 0.174 & 0.111 \\
\hline 15 & $A_{15}$ & 0.220 & 0.287 & 0.220 & 0.347 & 0.270 & 0.270 \\
\hline 16 & $x_{2}$ & 114.9670 & 101.5775 & 106.052 & 102.287 & 137.222 & 100.009 \\
\hline 17 & $x_{3}$ & 247.0400 & 227.9112 & 239.025 & 240.505 & 259.909 & 248.078 \\
\hline 18 & $y_{2}$ & 125.9190 & 134.7986 & 130.356 & 112.584 & 123.501 & 131.524 \\
\hline 19 & $y_{3}$ & 111.0670 & 128.2206 & 114.273 & 108.043 & 110.002 & 123.211 \\
\hline 20 & $y_{4}$ & 58.2980 & 54.8630 & 51.987 & 57.795 & 59.936 & 54.077 \\
\hline 21 & $y_{6}$ & -17.5640 & -16.4484 & 1.814 & -6.430 & -5.180 & -9.039 \\
\hline 22 & $y_{7}$ & -5.8210 & -13.3007 & 9.183 & -1.801 & 4.219 & -14.905 \\
\hline 23 & $y_{8}$ & 31.4650 & 54.8572 & 46.909 & 57.799 & 57.883 & 54.084 \\
\hline & ight (lb) & 75.5473 & 76.6854 & 82.2344 & 77.6153 & 72.6153 & 74.1673 \\
\hline & placement (in.) & 24.9993 & 24.9992 & 24.9999 & 24.9909 & 24.9912 & 24.9999 \\
\hline & uctural analyses & 8,000 & 8,000 & 4,500 & 4,500 & 4,500 & 4,000 \\
\hline
\end{tabular}

Table 3 compares the best design found by this work with those reported in the literature. It can be seen that the results achieved by the proposed algorithm are quite close to the best results reported in the literature.

4.2. Spatial 25-Bar Truss. The original geometry of spatial 25bar truss is shown in Figure 3(a). Two nodes (ID: 1 and 2) are totally fixed and the $z$-coordinates of four nodes (ID: 7, 8, 9. and 10) are fixed as well. The loading condition is listed in Table 4

To ensure the structural symmetries, all design variables are classified into 13 groups:

sizing variables: $A_{1} ; A_{2}=A_{3}=A_{4}=A_{5} ; A_{6}=A_{7}=$ $A_{8}=A_{9} ; A_{10}=A_{11} ; A_{12}=A_{13} ; A_{14}=A_{15}=A_{16}=$ $A_{17} ; A_{18}=A_{19}=A_{20}=A_{21} ; A_{22}=A_{23}=A_{24}=$ $A_{25}$; 


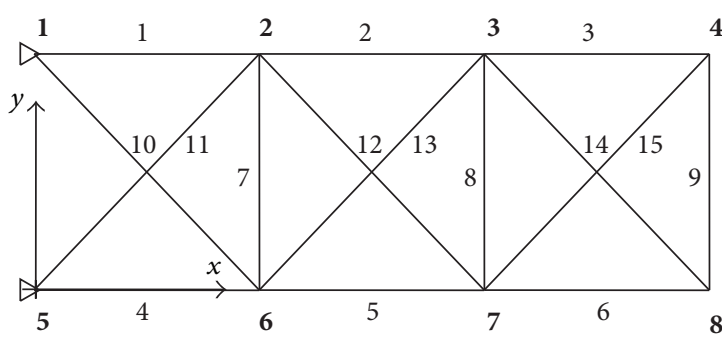

(a) Geometry and element definitions of the planar 15-bar truss

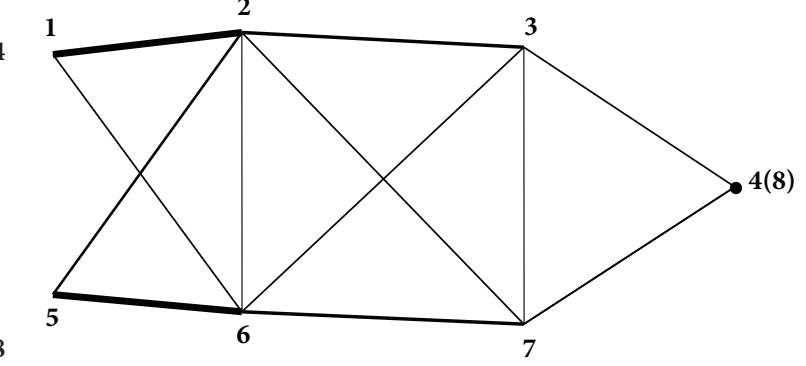

(b) Best solution of the planar 15-bar truss

FIgURE 2: Layout optimization of the planar 15-bar truss.

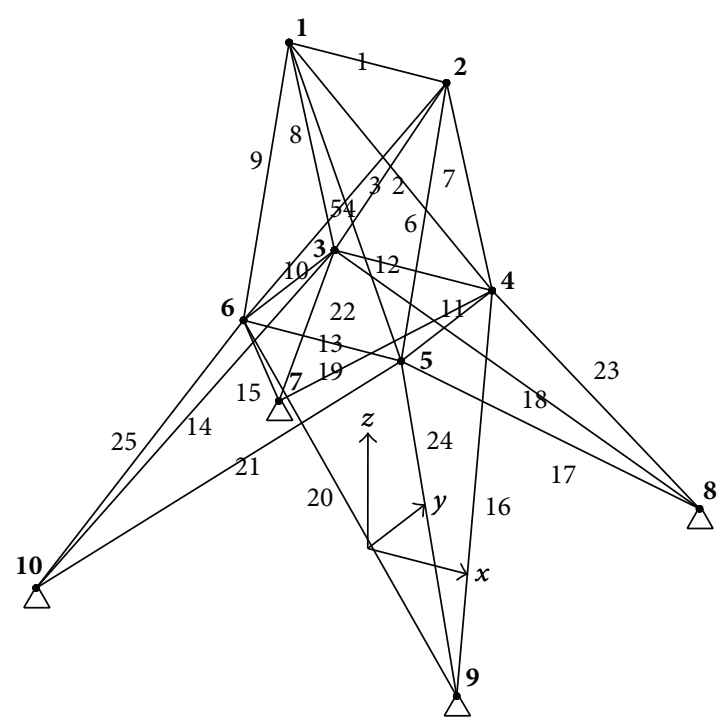

(a) Geometry and element definitions of the spatial 25-bar truss

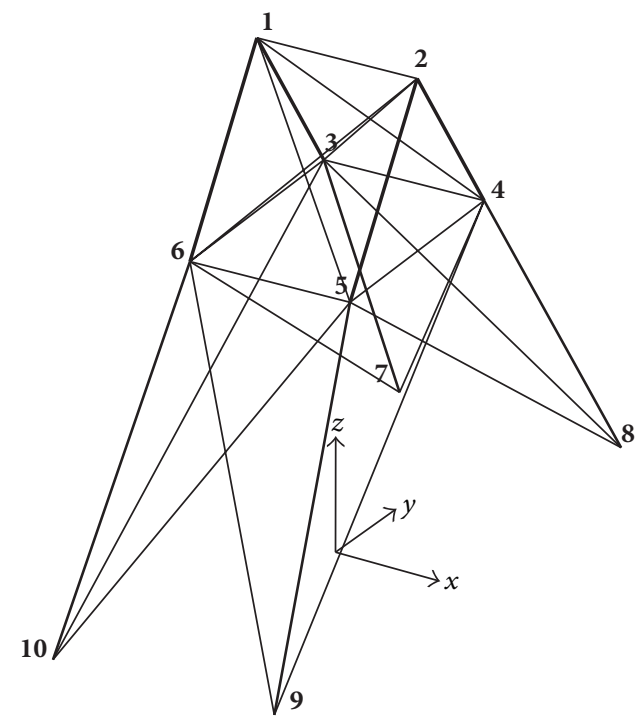

(b) Best solution of the spatial 25-bar truss

FIGURE 3: Layout optimization of the spatial 25-bar truss.

TABLE 4: Loading condition acting on the spatial 25-bar truss.

\begin{tabular}{ccccc}
\hline Case & Node & $F_{x}$ (kips) & $F_{y}$ (kips) & $F_{z}$ (kips) \\
\hline \multirow{3}{*}{1} & 1 & 1.0 & -10.0 & -10.0 \\
& 2 & 0.0 & -10.0 & -10.0 \\
& 3 & 0.5 & 0.0 & 0.0 \\
& 6 & 0.6 & 0.0 & 0.0 \\
\hline
\end{tabular}

shape variables: $x_{4}=x_{5}=-x_{3}=-x_{6} ; x_{8}=x_{9}=$ $-x_{7}=-x_{10} ; y_{3}=y_{4}=-y_{5}=-y_{6} ; y_{7}=y_{8}=-y_{9}=$ $-y_{10} ; z_{3}=z_{4}=z_{5}=z_{6}$.

Material parameters and design constraints are listed in Table 5.

Figure 3(b) shows the optimum design found by this work. Table 6 compares the best design found by this work with those reported in the literature. It can be concluded that by using the proposed algorithm it is possible to achieve the best feasible results at a low computational cost.
4.3. Planar 37-Bar Truss. The original geometry of planar 37bar truss is shown in Figure 4(a). Eleven nodes (ID: 1, 2, 4, 6, 8, $10,12,14,16,18$, and 20 ) are totally fixed and the $x$-coordinates of all other nine nodes (ID: 3, 5, 7, 9, 11, 13, 15, 17, and 19) are fixed as well. A nonstructural mass of $10 \mathrm{~kg}$ is attached to all free nodes on the lower chord.

To ensure the structural symmetric about the $y$-axis, all design variables are classified into 19 groups:

$$
\begin{aligned}
& \text { sizing variables: } A_{1}=A_{27} ; A_{2}=A_{26} ; A_{3}=A_{24} ; \\
& A_{4}=A_{25} ; A_{5}=A_{23} ; A_{6}=A_{21} ; A_{7}=A_{22} ; A_{8}= \\
& A_{20} ; A_{9}=A_{18} ; A_{10}=A_{19} ; A_{11}=A_{17} ; A_{12}=A_{15} ; \\
& A_{13}=A_{16} ; A_{14} ; \\
& \text { shape variables: } x_{3} ; x_{5} ; x_{7} ; x_{9} ; y_{3}=y_{19} ; y_{5}=y_{17} ; \\
& y_{7}=y_{15} ; y_{9}=y_{13} ; y_{11} .
\end{aligned}
$$

Material parameters and design constraints are listed in Table 7.

Figure 4(b) shows the optimum design explored by this work. Table 8 compares the best design presented by this work with those reported in the literature. It can be seen that 
TABLE 5: Material parameters, design constraints, and search range of the spatial 25-bar truss optimization problem.

\begin{tabular}{|c|c|}
\hline Category & Values \\
\hline \multicolumn{2}{|l|}{ Material parameters } \\
\hline Density & $0.1 \mathrm{lb} / \mathrm{in}^{3}$ \\
\hline Modulus of elasticity & $1 \times 10^{4} \mathrm{ksi}$ \\
\hline \multicolumn{2}{|l|}{ Constraints } \\
\hline Stress & The allowable elements stress interval: [ $-40 \mathrm{ksi}, 40 \mathrm{ksi}]$ \\
\hline Displacement & The allowable nodal displacement interval: [ -0.35 in., 0.35 in.] \\
\hline \multicolumn{2}{|l|}{ Search range } \\
\hline Shape variables & $\begin{array}{l}20 \text { in. } \leq x_{4} \leq 60 \text { in.; } 40 \text { in. } \leq x_{8} \leq 80 \text { in.; } 40 \text { in. } \leq y_{4} \leq 80 \text { in.; } 100 \text { in. } \leq y_{8} \leq 140 \text { in.; } 90 \text { in. } \leq z_{4} \leq 130 \text { in.; }-x_{1}= \\
x_{2}=37.5 \text { in.; } y_{1,2}=0 \text { in.; } z_{1,2}=200 \text { in. }\end{array}$ \\
\hline Sizing variables & $S=\{0.1,0.2,0.3, \ldots, 3.2,3.3,3.4\}$ in. $^{2} \quad A_{i} \in S, \quad i=1,2, \ldots, 25$ \\
\hline
\end{tabular}

TABLE 6: Comparison of optimized designs found for the spatial 25-bar truss.

\begin{tabular}{|c|c|c|c|c|c|c|c|}
\hline No. & Variable & FA [15] & FM-GA [16] & PSO [17] & CPSO [17] & SCPSO [17] & FEPSO-AP \\
\hline 1 & $A_{1}$ & 0.1 & 0.1 & 0.1 & 0.3 & 0.1 & 0.1 \\
\hline 2 & $A_{2}$ & 0.1 & 0.1 & 0.1 & 0.1 & 0.1 & 0.1 \\
\hline 3 & $A_{6}$ & 0.9 & 1.1 & 1.1 & 1.0 & 1.0 & 1.0 \\
\hline 4 & $A_{10}$ & 0.1 & 0.1 & 0.1 & 0.1 & 0.1 & 0.1 \\
\hline 5 & $A_{12}$ & 0.1 & 0.1 & 0.4 & 0.1 & 0.1 & 0.1 \\
\hline 6 & $A_{14}$ & 0.1 & 0.1 & 0.1 & 0.1 & 0.1 & 0.1 \\
\hline 7 & $A_{18}$ & 0.1 & 0.2 & 0.4 & 0.2 & 0.1 & 0.1 \\
\hline 8 & $A_{22}$ & 1.0 & 0.8 & 0.7 & 0.9 & 0.9 & 0.9 \\
\hline 9 & $x_{4}$ & 37.3200 & 33.0487 & 27.6169 & 33.4976 & 36.9520 & 36.8958 \\
\hline 10 & $y_{4}$ & 55.7400 & 53.5663 & 51.6196 & 62.3735 & 54.5786 & 54.1337 \\
\hline 11 & $z_{4}$ & 126.6200 & 129.9092 & 129.9071 & 114.5945 & 129.9758 & 130.0000 \\
\hline 12 & $x_{8}$ & 50.1400 & 43.7826 & 42.5526 & 40.0531 & 51.7317 & 51.9924 \\
\hline 13 & $y_{8}$ & 136.4000 & 136.8381 & 132.7241 & 133.6695 & 139.5316 & 140.0000 \\
\hline & eight (lb) & 118.83 & 120.1149 & 129.2076 & 123.5403 & 117.2271 & 117.3022 \\
\hline & splacement (in.) & 0.3500 & 0.3500 & 0.3503 & 0.3505 & 0.3518 & 0.3500 \\
\hline & stress (ksi) & 18.8302 & 17.1574 & 16.4391 & 15.5913 & 19.9702 & 20.0182 \\
\hline & stress (ksi) & -9.4017 & -6.4822 & -10.9931 & -6.4102 & -9.4049 & -9.4024 \\
\hline & uctural analyses & 6,000 & 10,000 & 4,500 & 4,500 & 4,500 & 4,500 \\
\hline
\end{tabular}

TABLE 7: Material parameters, design constraints, and search range of the planar 37-bar truss optimization problem.

\begin{tabular}{|c|c|}
\hline Category & Values \\
\hline \multicolumn{2}{|l|}{ Material parameters } \\
\hline Density & $7800 \mathrm{~kg} / \mathrm{m}^{3}$ \\
\hline Modulus of elasticity & $2.1 \times 10^{11} \mathrm{~N} / \mathrm{m}^{2}$ \\
\hline \multicolumn{2}{|l|}{ Constraints } \\
\hline Natural frequencies & $f_{1} \geq 20 \mathrm{~Hz} ; f_{2} \geq 40 \mathrm{~Hz} ; f_{3} \geq 60 \mathrm{~Hz}$ \\
\hline \multicolumn{2}{|l|}{ Search range } \\
\hline Shape variables & $\begin{array}{l}x_{1}=0 \mathrm{~m} ; x_{2,3}=1 \mathrm{~m} ; x_{4,5}=2 \mathrm{~m} ; x_{6,7}=3 \mathrm{~m} ; x_{8,9}=4 \mathrm{~m} ; x_{10,11}=5 \mathrm{~m} ; x_{12,13}= \\
6 \mathrm{~m} ; x_{14,15}=7 \mathrm{~m} ; \\
x_{16,17}=8 \mathrm{~m} ; x_{18,19}=9 \mathrm{~m} ; x_{20}=10 \mathrm{~m} ; 0 \mathrm{~m} \leq y_{3,5,7,9,11} \leq 3 \mathrm{~m} ; \\
1 \times 10^{-4} \mathrm{~m}^{2} \leq A_{i} \leq 10 \times 10^{-4} \mathrm{~m}^{2}, i=1,2, \ldots, 14 \\
A_{j}=4 \times 10^{-3} \mathrm{~m}^{2}, i=28,29, \ldots, 30\end{array}$ \\
\hline
\end{tabular}


TABLE 8: Comparison of optimized designs found for the planar 37-bar truss.

\begin{tabular}{|c|c|c|c|c|c|c|}
\hline No. & Variable & OC [9] & GA [18] & PSO [19] & $\mathrm{RO}[20]$ & FEPSO-AP \\
\hline 1 & $A_{1}$ & 3.2508 & 2.8932 & 2.6797 & 3.0124 & 3.4197 \\
\hline 2 & $A_{2}$ & 1.2364 & 1.1201 & 1.1568 & 1.0623 & 0.9766 \\
\hline 3 & $A_{3}$ & 1.0000 & 1.0000 & 2.3476 & 1.0005 & 0.8313 \\
\hline 4 & $A_{4}$ & 2.5386 & 1.8655 & 1.7182 & 2.2647 & 2.8073 \\
\hline 5 & $A_{5}$ & 1.3714 & 1.5962 & 1.2751 & 1.6339 & 1.2997 \\
\hline 6 & $A_{6}$ & 1.3681 & 1.2642 & 1.4819 & 1.6717 & 1.6483 \\
\hline 7 & $A_{7}$ & 2.4290 & 1.8254 & 4.6850 & 2.0591 & 2.4972 \\
\hline 8 & $A_{8}$ & 1.6522 & 2.0009 & 1.1246 & 1.6607 & 1.5379 \\
\hline 9 & $A_{9}$ & 1.8257 & 1.9526 & 2.1214 & 1.4941 & 1.7590 \\
\hline 10 & $A_{10}$ & 2.3022 & 1.9705 & 3.8600 & 2.4737 & 2.7069 \\
\hline 11 & $A_{11}$ & 1.3103 & 1.8294 & 2.9817 & 1.5260 & 1.3046 \\
\hline 12 & $A_{12}$ & 1.4067 & 1.2358 & 1.2021 & 1.4823 & 1.4004 \\
\hline 13 & $A_{13}$ & 2.1896 & 1.4049 & 1.2563 & 2.4148 & 3.0476 \\
\hline 14 & $A_{14}$ & 1.0000 & 1.0000 & 3.3276 & 1.0034 & 0.5947 \\
\hline 15 & $y_{3}$ & 1.2086 & 1.1998 & 0.9637 & 1.0010 & 0.8756 \\
\hline 16 & $y_{5}$ & 1.5788 & 1.6553 & 1.3978 & 1.3909 & 1.2546 \\
\hline 17 & $y_{7}$ & 1.6719 & 1.9652 & 1.5929 & 1.5893 & 1.4446 \\
\hline 18 & $y_{9}$ & 1.7703 & 2.0737 & 1.8812 & 1.7507 & 1.5889 \\
\hline 19 & $y_{11}$ & 1.8502 & 2.3050 & 2.0856 & 1.8336 & 1.6480 \\
\hline & $f_{1}$ & 20.0850 & 20.0013 & 20.0001 & 20.056 & 20.020 \\
\hline & $f_{2}$ & 42.0743 & 40.0305 & 40.0003 & 40.035 & 40.022 \\
\hline Natural frequencies $(\mathrm{Hz})$ & $f_{3}$ & 62.9383 & 60.0000 & 60.0001 & 60.030 & 60.233 \\
\hline & $f_{4}$ & 74.4539 & 73.0444 & 73.0440 & 74.387 & 72.137 \\
\hline & $f_{5}$ & 90.0576 & 89.8244 & 89.8240 & 85.929 & 84.065 \\
\hline Best weight & & 366.50 & 368.84 & 377.20 & 364.04 & 362.8812 \\
\hline \multicolumn{2}{|c|}{ Number of structural analyses } & - & - & 20,000 & 32,000 & 8,000 \\
\hline
\end{tabular}

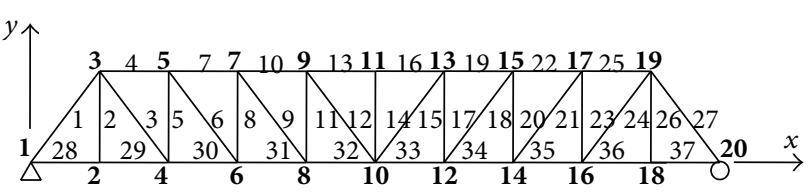

(a) Geometry and element definitions of the planar 37-bar truss

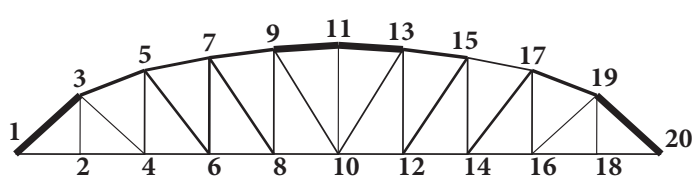

(b) Best solution of the planar 37-bar truss

FIGURE 4: Layout optimization of the planar 37-bar truss.

TABLE 9: Loading conditions acting on the planar 47-bar truss.

\begin{tabular}{lccc}
\hline Case & Node & $F_{x}$ (kips) & $F_{y}$ (kips) \\
\hline 1 & 17 and 22 & 6.0 & -14.0 \\
2 & 17 & 6.0 & -14.0 \\
3 & 22 & 6.0 & -14.0 \\
\hline
\end{tabular}

the results achieved by the proposed algorithm are even better than the best results reported in the literature.

4.4. Planar 47-Bar Truss. The original geometry of planar 47bar truss is shown in Figure 5(a). Four nodes (ID: 15, 16, 17, and 22) are totally fixed and the $y$-coordinates of other two nodes (ID: 1 and 2) are fixed as well. The loading conditions are listed in Table 9.
To ensure the structural symmetric about the $y$-axis, all design variables are classified into 44 groups:

$$
\begin{aligned}
& \text { sizing variables: } A_{1}=A_{3} ; A_{2}=A_{4} ; A_{5}=A_{6} ; A_{7} ; \\
& A_{8}=A_{9} ; A_{10} ; A_{11}=A_{12} ; A_{13}=A_{14} ; A_{15}=A_{16} ; \\
& A_{17}=A_{18} ; A_{19}=A_{20} ; A_{21}=A_{22} ; A_{23}=A_{24} ; A_{25}= \\
& A_{26} ; A_{27} ; A_{28} ; A_{29}=A_{30} ; A_{31}=A_{32} ; A_{33} ; A_{34}= \\
& A_{35} ; A_{36}=A_{37} ; A_{38} ; A_{39}=A_{40} ; A_{41}=A_{42} ; A_{43} ; \\
& A_{44}=A_{45} ; A_{46}=A_{47} ; \\
& \text { shape variables: } x_{1}=-x_{2} ; x_{3}=-x_{4} ; y_{3}=y_{4} ; x_{5}= \\
& -x_{6} ; y_{5}=y_{6} ; x_{7}=-x_{8} ; y_{7}=y_{8} ; x_{9}=-x_{10} ; y_{9}=y_{10} ; \\
& x_{11}=-x_{12} ; y_{11}=y_{12} ; x_{13}=-x_{14} ; y_{13}=y_{14} ; x_{19}= \\
& -x_{20} ; y_{19}=y_{20} ; x_{18}=-x_{21} ; y_{18}=y_{21} .
\end{aligned}
$$

Material parameters and design constraints are listed in Table 10. 
TABLE 10: Material parameters, design constraints, and search range of the planar 47-bar truss optimization problem.

\begin{tabular}{|c|c|}
\hline Category & Values \\
\hline \multicolumn{2}{|l|}{ Material parameters } \\
\hline Density & $0.3 \mathrm{lb} / \mathrm{in}^{3}$ \\
\hline Modulus of elasticity & $3 \times 10^{4} \mathrm{ksi}$ \\
\hline \multicolumn{2}{|l|}{ Constraints } \\
\hline Stress & The allowable elements stress interval: [ $-15 \mathrm{ksi}, 20 \mathrm{ksi}]$ \\
\hline Local buckling & $\left|\left(\sigma_{c}\right)_{i}\right| \leq \beta E A_{i} / l_{i}^{2}, \quad i=1,2, \ldots, 47, \quad \beta=3.96$ \\
\hline \multicolumn{2}{|l|}{ Search range } \\
\hline Shape variables & $\begin{array}{l}0 \text { in. } \leq x_{i} \leq 120 \text { in., }(i=2,4,6,8) ;-30 \text { in. } \leq x_{j} \leq 90 \text { in. }(j=10,12,14,20) ; 30 \text { in. } \leq x_{21} \leq 150 \text { in.; } \\
60 \text { in. } \leq y_{4} \leq 180 \text { in.; } 180 \text { in. } \leq y_{6} \leq 300 \text { in.; } 300 \text { in. } \leq y_{8} \leq 420 \text { in.; } 360 \text { in. } \leq y_{10} \leq 480 \text { in.; } 420 \text { in. } \\
\leq y_{12} \leq 540 \text { in.; } 480 \text { in. } \leq y_{14} \leq 600 \text { in.; } 540 \text { in. } \leq y_{20} \leq 660 \text { in.; } 540 \text { in. } \leq y_{21} \leq 660 \text { in. }\end{array}$ \\
\hline Sizing variables & $S=\{0.1,0.2,0.3, \ldots, 4.8,4.9,5.0\}$ in. $^{2} \quad A_{i} \in S, \quad i=1,2, \ldots, 47$ \\
\hline
\end{tabular}

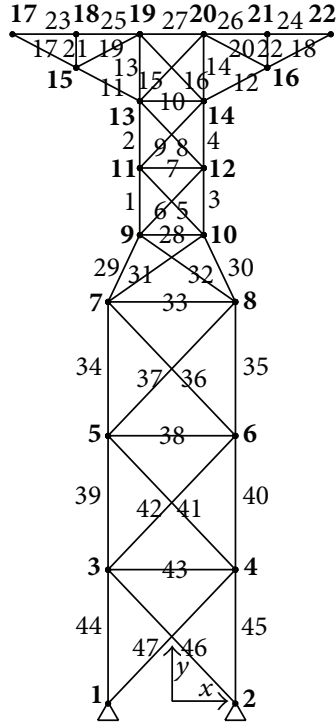

(a) Geometry and element definitions of the planar 47 bar truss

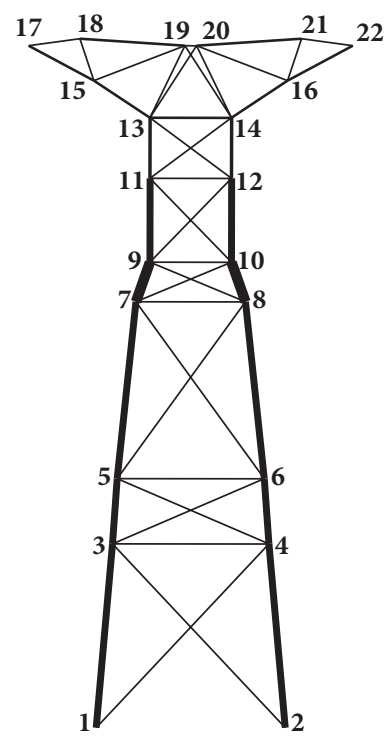

(b) Best solution of the planar 47bar truss

FIGURE 5: Layout optimization of the planar 47-bar truss.

Figure 5(b) shows the optimum design identified by this work. Table 11 compares the best design of this work with those reported in the literature. It can be seen that by using the proposed algorithm it is possible to achieve better results at a lower computational cost.

\section{Conclusion}

In this work, a new hybrid PSO algorithm is proposed to solve a quite challenging task in truss optimization area: truss layout optimization with multiple constraints.

Two computational techniques are adopted to further enhance the performance of PSO algorithm. In the first fitness estimation strategy, the evaluation of particles is partly substituted by the estimation of similar particles, with the purpose to reduce the computational cost of real world optimization problem. In the second adaptive penalty function approach, the iteration information is merged into the penalty function to find a good balance between the exploration and exploitation of the constrained design domain. The resulted algorithm is termed as FEPSO-AP.

Four benchmark truss layout optimization problems, subject to nodal displacement constraints, element stress constraints, natural frequency constraints, and local buckling constraints, are used to verify the performance of FEPSOAP. Numerical results demonstrate that three out of four benchmarks, to which the FEPSO-AP based optimization is applied, delivered the best feasible designs to the author's knowledge. Moreover, the convergence rate of the FEPSO-AP algorithm is quite competitive comparing to other state-ofthe-art hybrid algorithms published in the former literatures.

\section{Conflict of Interests}

The authors declare that there is no conflict of interests regarding the publication of this paper. 
TABLE 11: Comparison of optimized designs found for the planar 47-bar truss.

\begin{tabular}{|c|c|c|c|c|c|c|c|}
\hline No. & Variable & GA [21] & FSD-ES [22] & PSO [17] & CPSO [17] & SCPSO [17] & FEPSO-AP \\
\hline 1 & $A_{3}$ & 2.50 & 2.70 & 2.80 & 2.60 & 2.50 & 3.20 \\
\hline 2 & $A_{4}$ & 2.20 & 2.50 & 2.70 & 2.50 & 2.50 & 2.80 \\
\hline 3 & $A_{5}$ & 0.70 & 0.70 & 0.80 & 0.70 & 0.80 & 0.70 \\
\hline 4 & $A_{7}$ & 0.10 & 0.10 & 1.10 & 0.30 & 0.10 & 0.10 \\
\hline 5 & $A_{8}$ & 1.30 & 0.90 & 0.80 & 1.20 & 0.70 & 0.60 \\
\hline 6 & $A_{10}$ & 1.30 & 1.10 & 1.30 & 1.10 & 1.40 & 1.60 \\
\hline 7 & $A_{12}$ & 1.80 & 1.80 & 1.80 & 1.60 & 1.70 & 1.90 \\
\hline 8 & $A_{14}$ & 0.50 & 0.70 & 0.90 & 0.80 & 0.80 & 0.90 \\
\hline 9 & $A_{15}$ & 0.80 & 0.90 & 1.20 & 1.10 & 0.90 & 1.00 \\
\hline 10 & $A_{18}$ & 1.20 & 1.30 & 1.40 & 1.30 & 1.30 & 2.00 \\
\hline 11 & $A_{20}$ & 0.40 & 0.30 & 0.30 & 0.30 & 0.30 & 0.10 \\
\hline 12 & $A_{22}$ & 1.20 & 1.10 & 1.40 & 0.80 & 0.90 & 0.40 \\
\hline 13 & $A_{24}$ & 0.90 & 1.00 & 1.10 & 1.00 & 1.00 & 1.40 \\
\hline 14 & $A_{26}$ & 1.00 & 0.90 & 1.20 & 1.00 & 1.10 & 1.50 \\
\hline 15 & $A_{27}$ & 3.60 & 0.80 & 1.60 & 0.90 & 5.00 & 1.20 \\
\hline 16 & $A_{28}$ & 0.10 & 0.10 & 1.00 & 0.10 & 0.10 & 0.70 \\
\hline 17 & $A_{30}$ & 2.40 & 2.70 & 2.80 & 2.70 & 2.50 & 5.00 \\
\hline 18 & $A_{31}$ & 1.10 & 0.80 & 0.80 & 0.90 & 1.00 & 1.00 \\
\hline 19 & $A_{33}$ & 0.10 & 0.10 & 0.10 & 0.10 & 0.10 & 0.10 \\
\hline 20 & $A_{35}$ & 2.70 & 3.00 & 3.00 & 3.00 & 2.80 & 3.00 \\
\hline 21 & $A_{36}$ & 0.80 & 0.90 & 0.90 & 1.00 & 0.90 & 0.50 \\
\hline 22 & $A_{38}$ & 0.10 & 0.00 & 0.10 & 0.20 & 0.10 & 0.10 \\
\hline 23 & $A_{40}$ & 2.80 & 3.20 & 3.30 & 3.30 & 3.00 & 3.30 \\
\hline 24 & $A_{41}$ & 1.30 & 1.00 & 0.90 & 0.90 & 1.00 & 0.30 \\
\hline 25 & $A_{43}$ & 0.20 & 0.10 & 0.10 & 0.10 & 0.10 & 0.10 \\
\hline 26 & $A_{45}$ & 3.00 & 3.30 & 3.30 & 3.30 & 3.20 & 3.50 \\
\hline 27 & $A_{46}$ & 1.20 & 1.10 & 1.20 & 1.10 & 1.20 & 0.40 \\
\hline 28 & $x_{2}$ & 114.0000 & 100.9724 & 98.8628 & 99.3630 & 101.3393 & 87.7275 \\
\hline 29 & $x_{4}$ & 97.0000 & 80.4772 & 78.6595 & 83.4439 & 85.9111 & 72.4352 \\
\hline 30 & $y_{4}$ & 125.0000 & 136.8699 & 146.7331 & 126.3845 & 135.9645 & 162.6451 \\
\hline 31 & $x_{6}$ & 76.0000 & 64.3908 & 66.5231 & 69.5148 & 74.7969 & 67.2113 \\
\hline 32 & $y_{6}$ & 261.0000 & 247.0491 & 239.0901 & 218.2013 & 237.7447 & 218.2041 \\
\hline 33 & $x_{8}$ & 69.0000 & 55.2589 & 55.6936 & 58.0004 & 64.3115 & 50.6507 \\
\hline 34 & $y_{8}$ & 316.0000 & 338.4534 & 327.7882 & 322.2272 & 321.3416 & 375.4549 \\
\hline 35 & $x_{10}$ & 56.0000 & 48.7333 & 48.8641 & 51.4015 & 53.3345 & 36.6525 \\
\hline 36 & $y_{10}$ & 414.0000 & 409.7380 & 398.6775 & 401.5626 & 414.3025 & 408.7230 \\
\hline 37 & $x_{12}$ & 50.0000 & 43.4742 & 43.1400 & 46.8605 & 46.0277 & 36.9960 \\
\hline 38 & $y_{12}$ & 463.0000 & 472.1479 & 464.7831 & 458.3021 & 489.9216 & 483.4295 \\
\hline 39 & $x_{14}$ & 54.0000 & 44.8349 & 37.8993 & 46.8885 & 41.8353 & 37.9558 \\
\hline 40 & $y_{14}$ & 524.0000 & 512.1901 & 511.0450 & 527.8575 & 522.4161 & 535.7644 \\
\hline 41 & $x_{20}$ & 1.0000 & 3.8414 & 18.2341 & 16.2354 & 1.0005 & 4.6875 \\
\hline 42 & $y_{20}$ & 587.0000 & 591.1449 & 594.0710 & 610.8496 & 598.3905 & 599.7416 \\
\hline 43 & $x_{21}$ & 99.0000 & 84.5040 & 90.9369 & 98.3239 & 97.8696 & 101.4535 \\
\hline 44 & $y_{21}$ & 631.0000 & 630.3472 & 621.3943 & 624.9580 & 624.0552 & 605.4302 \\
\hline & ight (lb) & 1925.7897 & 1842.6609 & 1975.8393 & 1908.8301 & 1864.0985 & 1799.7037 \\
\hline & stress (ksi) & 19.9528 & 20.0000 & 19.0636 & 19.3351 & 19.4735 & 19.9808 \\
\hline & stress (ksi) & -14.9973 & -15.0000 & -14.9999 & -14.9986 & -15.0000 & -14.9986 \\
\hline & ckling elements & 0 & 0 & 0 & 0 & 0 & 0 \\
\hline & uctural analyses & 100,000 & 55,802 & 25,000 & 25,000 & 25,000 & 20,000 \\
\hline
\end{tabular}




\section{References}

[1] S. Shan and G. G. Wang, "Survey of modeling and optimization strategies to solve high-dimensional design problems with computationally-expensive black-box functions," Structural and Multidisciplinary Optimization, vol. 41, no. 2, pp. 219-241, 2010.

[2] A. Kaveh and A. Zolghadr, "Democratic PSO for truss layout and size optimization with frequency constraints," Computers and Structures, vol. 130, pp. 10-21, 2014.

[3] A. Xiao, B. Wang, and Y. Jin, "Evolutionary truss layout optimization using the vectorized structure approach," in Proceedings of the IEEE Congress on Evolutionary Computation (CEC '13), pp. 2879-2886, June 2013.

[4] M. W. Dobbs and L. P. Felton, "Optimization of truss geometry," Journal of the Structural Division, vol. 95, pp. 2105-2118, 1969.

[5] G. N. Vanderplaats and F. Moses, "Structural optimization by methods of feasible directions," Computers and Structures, vol. 3, no. 4, pp. 739-755, 1973.

[6] M. Zhou, "Geometrical optimization of trusses by a two-level approximation concept," Structural Optimization, vol. 1, no. 4, pp. 235-240, 1989.

[7] L. Gil and A. Andreu, "Shape and cross-section optimization of a truss structure," Computers \& Structures, vol. 79, no. 7, pp. 681-689, 2001.

[8] K. Deb and S. Gulati, "Design of truss-structures for minimum weight using genetic algorithms," Finite Elements in Analysis and Design, vol. 37, no. 5, pp. 447-465, 2001.

[9] D. Wang, W. H. Zhang, and J. S. Jiang, "Truss optimization on shape and sizing with frequency constraints," AIAA Journal, vol. 42, no. 3, pp. 622-630, 2004.

[10] P. C. Fourie and A. A. Groenwold, "The particle swarm optimization algorithm in size and shape optimization," Structural and Multidisciplinary Optimization, vol. 23, no. 4, pp. 259-267, 2002.

[11] O. Hasançebi and F. Erbatur, "Layout optimisation of trusses using simulated annealing," Advances in Engineering Software, vol. 33, no. 7-10, pp. 681-696, 2002.

[12] H. Kawamura, H. Ohmori, and N. Kito, "Truss topology optimization by a modified genetic algorithm," Structural and Multidisciplinary Optimization, vol. 23, no. 6, pp. 467-472, 2002.

[13] A. Kaveh and S. Talatahari, "Optimal design of skeletal structures via the charged system search algorithm," Structural and Multidisciplinary Optimization, vol. 41, no. 6, pp. 893-911, 2010.

[14] M. Sonmez, "Artificial Bee Colony algorithm for optimization of truss structures," Applied Soft Computing Journal, vol. 11, no. 2, pp. 2406-2418, 2011.

[15] L. F. F. Miguel, R. H. Lopez, and L. F. F. Miguel, "Multimodal size, shape, and topology optimisation of truss structures using the Firefly algorithm," Advances in Engineering Software, vol. 56, pp. 23-37, 2013.

[16] H. Rahami, A. Kaveh, and Y. Gholipour, "Sizing, geometry and topology optimization of trusses via force method and genetic algorithm," Engineering Structures, vol. 30, no. 9, pp. 2360-2369, 2008.

[17] S. Gholizadeh, "Layout optimization of truss structures by hybridizing cellular automata and particle swarm optimization," Computers and Structures, vol. 125, pp. 86-99, 2013.

[18] W. Lingyun, Z. Mei, W. Guangming, and M. Guang, "Truss optimization on shape and sizing with frequency constraints based on genetic algorithm," Computational Mechanics, vol. 35, no. 5, pp. 361-368, 2005.
[19] H. M. Gomes, "Truss optimization with dynamic constraints using a particle swarm algorithm," Expert Systems with Applications, vol. 38, no. 1, pp. 957-968, 2011.

[20] A. Kaveh and M. Khayatazad, "Ray optimization for size and shape optimization of truss structures," Computers and Structures, vol. 117, pp. 82-94, 2013.

[21] O. Hasançebi and F. Erbatur, "Layout optimization of trusses using improved GA methodologies," Acta Mechanica, vol. 146, no. 1-2, pp. 87-107, 2001.

[22] A. Ahrari, A. A. Atai, and K. Deb, "Simultaneous topology, shape and size optimization of truss structures by fully stressed design based on evolution strategy," Engineering Optimization, 2013.

[23] A. Kaveh and A. Zolghadr, "Truss optimization with natural frequency constraints using a hybridized CSS-BBBC algorithm with trap recognition capability," Computers \& Structures, vol. 102-103, pp. 14-27, 2012.

[24] W. Zuo, J. Bai, and B. Li, "A hybrid OC-GA approach for fast and global truss optimization with frequency constraints," Applied Soft Computing Journal, vol. 14, pp. 528-535, 2014.

[25] A. Kaveh and S. M. Javadi, "Shape and size optimization of trusses with multiple frequency constraints using harmony search and ray optimizer for enhancing the particle swarm optimization algorithm," Acta Mechanica, vol. 225, no. 6, pp. 1595-1605, 2014.

[26] W. Liu and J. Ye, "Collapse optimization for domes under earthquake using a genetic simulated annealing algorithm," Journal of Constructional Steel Research, vol. 97, pp. 59-68, 2014.

[27] R. Eberhart and J. Kennedy, "A new optimizer using particle swarm theory," in Proceedings of the 6th International Symposium on Micro Machine and Human Science, vol. 1, pp. 39-43, October 1995.

[28] C. Sun, J. Zeng, J. Pan, S. Xue, and Y. Jin, "A new fitness estimation strategy for particle swarm optimization," Information Sciences, vol. 221, pp. 355-370, 2013.

[29] C. A. Coello Coello, "Theoretical and numerical constrainthandling techniques used with evolutionary algorithms: a survey of the state of the art," Computer Methods in Applied Mechanics and Engineering, vol. 191, no. 11-12, pp. 1245-1287, 2002.

[30] T. P. Runarsson and X. Yao, "Stochastic ranking for constrained evolutionary optimization," IEEE Transactions on Evolutionary Computation, vol. 4, no. 3, pp. 284-294, 2000.

[31] M. Clerc and J. Kennedy, "The particle swarm-explosion, stability, and convergence in a multidimensional complex space," IEEE Transactions on Evolutionary Computation, vol. 6, no. 1, pp. 58-73, 2002. 


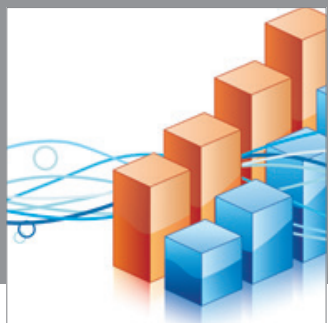

Advances in

Operations Research

mansans

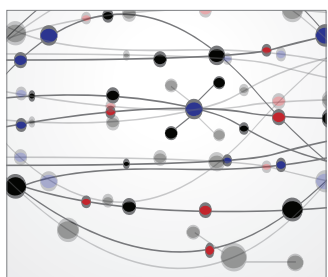

The Scientific World Journal
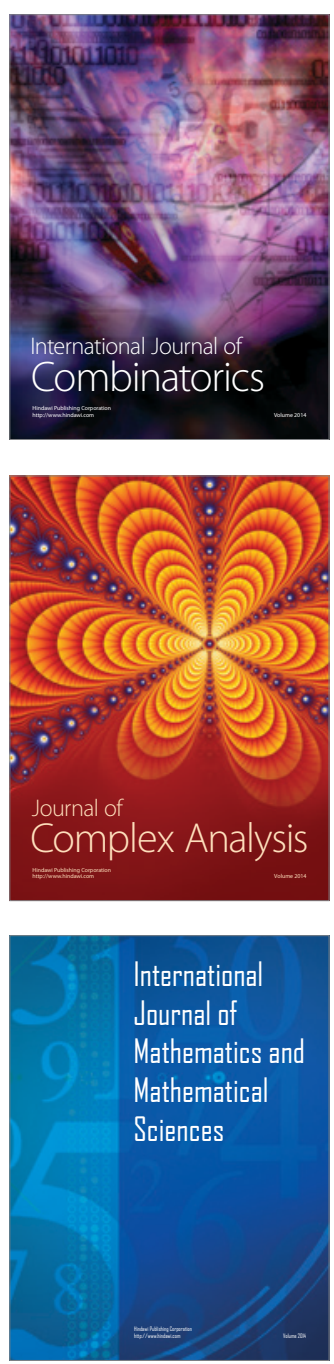
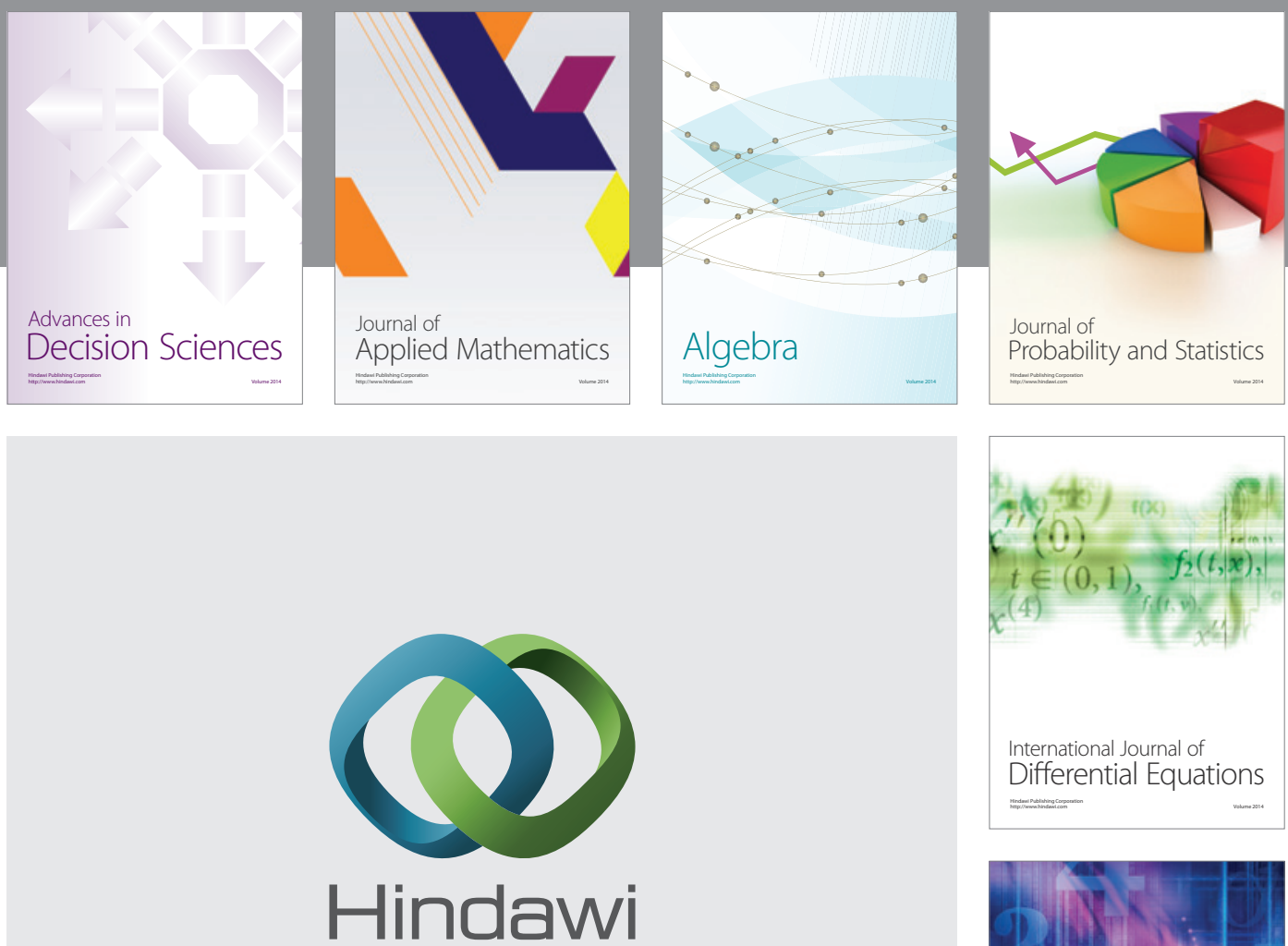

Submit your manuscripts at http://www.hindawi.com
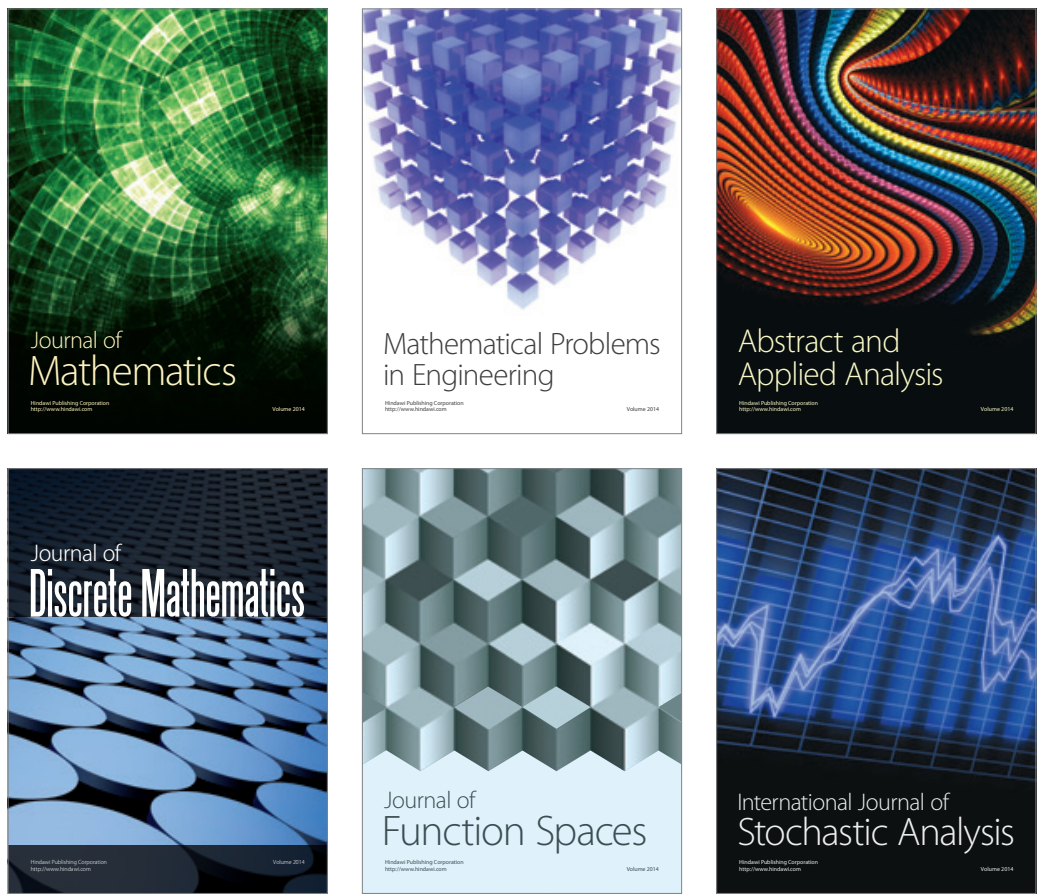

Journal of

Function Spaces

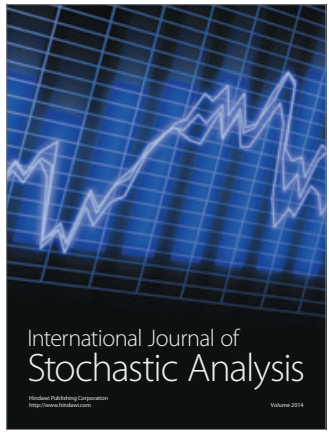

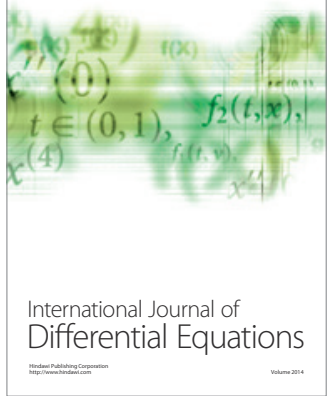
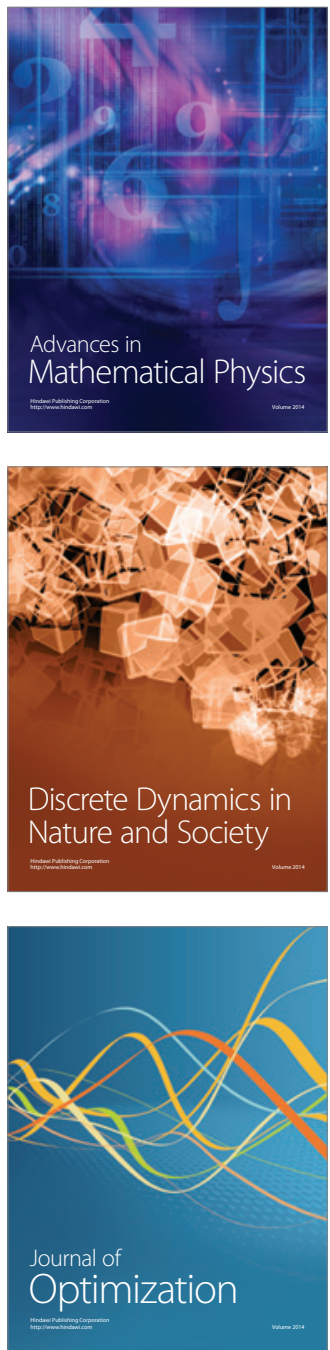